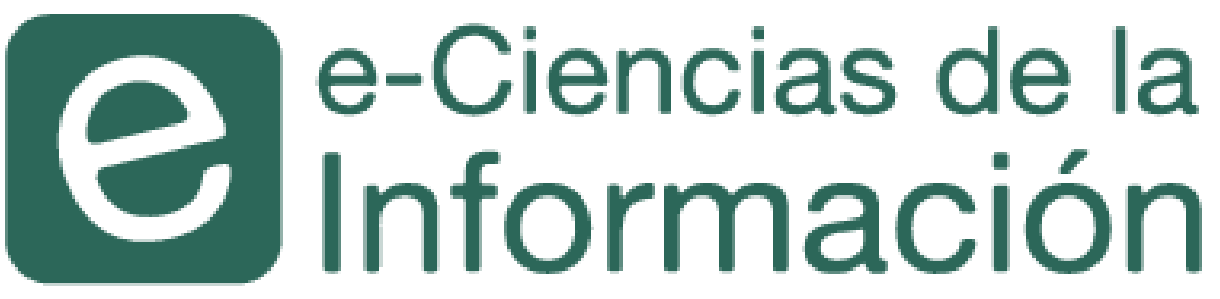

\title{
Modelo de sostenibilidad para Centros Comunitarios Inteligentes de Costa Rica
}

$>$ Dra. Susan Chen Mok Publicado 01 de enero, 2016 / Informe técnico 3 Revista electrónica semestral ISSN-1659-4142

\section{(ㄷ)(구(2)}

Escuela de Bibliotecología y Ciencias de la Información Universidad de Costa Rica

Visite el sitio web de e-Ciencias de la Información 


\title{
Modelo de sostenibilidad para Centros Comunitarios Inteligentes de Costa Rica
}

\author{
Sustainability Model for Intelligent Community Centers of Costa Rica \\ Dra. Susan Chen Mok'
}

\section{RESUMEN}

Este artículo presenta un modelo de trabajo que permitirá dar sostenibilidad a los Centros Comunitarios Inteligentes (CECI), con el fin de continuar con los procesos de apropiación de la tecnología por parte de las comunidades en donde se ubican, y por lo tanto disminuir la brecha digital. Antes de presentar el modelo, se ofrecen los antecedentes conceptuales que fundamentan el proyecto de CECI. Para la creación del modelo, en el 2014 se realizó un diagnóstico del funcionamiento y administración de algunos CECl existentes en el Cantón Central de Puntarenas, que se encuentran en un radio máximo de $12 \mathrm{~km}$ de la Sede de Pacífico de la Universidad de Costa Rica, y se indagó sobre los actores locales que podrían intervenir. Se considera que el modelo es replicable para todos los Centros Comunitarios Inteligentes existentes en todo el país, con la diferencia de las necesidades propias de cada comunidad en donde estos se encuentran.

\section{ABSTRACT}

This article presents a working model to allow the sustainability of Intelligent Community Centers (CECI); its purpose is continuing with the processes for the local communities to own the technology, and therefore reduce the digital gap. Before presenting the model, we provide the conceptual background that provides the basis for CECI. In order to develop the model, in 2014 we conducted a diagnosis of the operation and administration of some CECl which exist in the Central County of Puntarenas, that are located within a maximum of 12 $\mathrm{km}$ from the Pacific Campus of the University of Costa Rica, and we studied the local actors that could get involved in the model. We considered that it is possible to replicate the model across all $\mathrm{CECl}$ existing in the entire country, but considering the specific needs of the local communities where these centers are located.

\section{Palabras clave}

Centros Comunitarios Inteligentes, brecha digital, tecnologías de información y comunicación, educación, modelo de sostenibilidad, Costa Rica

\section{Keywords}

Intelligent Community Centers, digital gap, information and communication technologies, education, sustainability model, Costa Rica

Recibido: 19 de jul 2015 | Corregido: 25 de oct, 2015 | Aprobado: 27 de oct, 2015 


\section{Introducción}

El trabajo presenta los resultados del proyecto de investigación inscrito en la Universidad de Costa Rica (UCR) denominado 550-B4-009 Modelo de capacitación sostenible para Centros Comunitarios Inteligentes (CECI): estrategia para disminuir la brecha digital en Costa Rica. El objetivo de esta investigación es desarrollar un modelo que permita la sostenibilidad de los Centros Comunitarios Inteligentes, a partir del análisis y estudio del funcionamiento de algunos de estos, ubicados en los alrededores de la Sede del Pacífico de la UCR.

La investigación surge a partir de la indagación sobre el Programa de Gobierno Digital de Costa Rica y la necesidad de disminuir la brecha digital del país en aras de tener una ciudadanía capaz de enfrentar el desarrollo y utilizar las tecnologías de información y comunicación (TIC) para relacionarse con el Estado y atender sus propias necesidades de desarrollo para mejorar su calidad de vida.

El trabajo presenta algunos conceptos importantes alrededor del tema de brecha digital, seguidos del concepto y algunos datos sobre los $\mathrm{CECl}$, para luego informar sobre los resultados más importantes del diagnóstico realizado. Adicionalmente, indaga sobre los espacios de coordinación interinstitucional existentes en el cantón para concluir con una propuesta de modelo de sostenibilidad para estos Centros.

\section{Antecedentes y conceptos}

En la última década, toda la población ha percibido el vertiginoso desarrollo de las TIC. Un ejemplo de ello es la proliferación de herramientas tecnológicas como teléfonos celulares inteligentes, tabletas, televisores inteligentes, etc.

Por su parte, en Costa Rica, de un total de 1.399.271 viviendas, el 55\% tiene accesos a diversas tecnologías. De este total, 126.030 viviendas se encuentran en zonas rurales y 605.867 en la meseta central (Instituto Nacional de Estadística y Censos [INEC], 2014).

Solo en lo que se refiere a la telefonía celular, encontramos en INEC (2014) que las zonas rurales de Costa Rica tienen un porcentaje similar a la meseta central. Además, se observa que en lo referente al acceso a internet por dispositivos, la zona rural tiene un porcentaje mayor a la zona central.

Para julio del 2014, Lara (2014) informa que "el arribo de operadores telefónicos y el mayor dinamismo del mercado, se encargaron de poner Internet en manos de los costarricenses. Hoy, el $88 \%$ del acceso nacional a esa red se hace desde un teléfono celular" (p. 17A). Para el 2010 había 606.000 suscripciones al servicio de internet móvil, al finalizar el 2013, esta cifra creció a 3,5 millones; y si se incluye los enlaces fijos, esta cifra aumenta a 4 millones.

Para proseguir, diversos autores han definido de diferentes maneras el concepto de brecha digital, tomaremos una de ellas solo para tener una referencia de su significado: "la separación que existe entre las personas (comunidades, estados, países...) que utilizan las TIC como una 
parte rutinaria de su vida diaria y aquellas que no tienen acceso a las mismas y que aunque las tengan no saben cómo utilizarlas" (Serrano y Martínez, 2003, p. 8). ${ }^{2}$

Como puede observarse de estos datos, la mayoría de la población está accediendo a las TIC a través del uso de sus teléfonos móviles así como de Internet móvil. Sin embargo, como lo indica Fonseca (2012): "La adquisición de tecnología como celulares no significa que las personas la estén aprovechando en procesos educativos o productivos. Así, aunque se cierra una brecha de las herramientas, se abre una nueva la del conocimiento" (p.18 A).

Por otro lado, también tenemos el concepto de alfabetización digital:

La alfabetización digital tiene como objetivos proporcionar el conocimiento necesario para comprender los lenguajes de los documentos multimedia interactivos y el modo en que se integran, hacer uso de los dispositivos y técnicas en el procesamiento de la información, propiciar la valoración de las implicaciones socioculturales involucrados en las nuevas tecnologías multimedia, y favorecer la criticidad y responsabilidad en el contexto de la comunicación democrática en los medios multimedia ... La alfabetización digital es un fenómeno de gran importancia que se encuentra involucrado en la mejora de la calidad de vida, el desarrollo integral de las personas y el crecimiento socioeconómico de la sociedad, ya que le permite al individuo estar informado, tener más posibilidades de empleo y de recreación, así como tener una mayor participación y uso de las herramientas necesarias para los fines que desee, tanto a nivel personal como profesional. (Muñoz y Nicaragua, 2014, pp. 10-11)

El informe PROSIC (Programa Sociedad de la Información y el Conocimiento, 2006), presenta un capítulo dedicado a la Brecha Digital de Costa Rica, en el que se informa una serie de desigualdades existentes entre distintos niveles regionales y grupos humanos con relación al uso y aprovechamiento de las TIC. Este informe deja claro que el origen de la brecha se encuentra en las disparidades sociales de carácter estructural como la desigualdad en la distribución del ingreso, en la dotación selectiva de la infraestructura de comunicaciones y en el desigual nivel educativo de personas y comunidades. Deja claro que las repercusiones de la brecha digital inciden en la competitividad económica de los países creando nuevas formas de producción que excluyen a las personas que carecen de formación y habilidades en el campo de las TIC. Además, estas personas ven limitada su capacidad para participar activamente en procesos políticos y sociales a través de la red.

Como se ha mencionado, ya la población está teniendo acceso a las herramientas, pero ¿de qué manera se puede disminuir la brecha digital? ¿Cómo podemos realizar la alfabetización digital? Es decir, ¿cómo se puede lograr que la población utilice esas tecnologías en su propio beneficio, en procesos educativos, productivos y económicos que le generen bienestar y calidad de vida? En Costa Rica se identifican varios proyectos que trabajan el tema de brecha digital (Muñoz y Nicaragua, 2014), uno de los cuales es el proyecto de CECI que se describe a continuación. 


\section{Los Centros Comunitarios Inteligentes}

En Costa Rica, el Ministerio de Ciencia, Tecnología y Telecomunicaciones (MICITT) tiene un proyecto denominado Centros Comunitarios Inteligentes (CECI). Se trata de una red nacional compuesta de laboratorios equipados con computadoras de última tecnología que ofrecen diversos servicios a la comunidad. Mediante esta red de CECI se busca promover el desarrollo socioeconómico de las regiones por medio de la alfabetización digital, procurando el empoderamiento tecnológico de las comunidades al permitir el acceso al conocimiento, la información, la creatividad y la capacidad de asumir nuevos retos; además, en una próxima etapa, se buscará dar servicios a las pequeñas y medianas empresas (PyMES) y formar usuarios capaces de utilizar los servicios de la administración digital del Estado (MICITT, 2014a).

Los primeros CECl se instalaron en el 2007, para el 2010 se habían instalados 271 CECl. Desde su concepción en el 2006 hasta la actualidad, los CECI han tenido problemas en su funcionamiento y administración. Entre los problemas que han tenido están: falta de presupuesto y recursos para su atención, falta de un encargado del centro, falta de apropiación por parte de la comunidad, así como una entidad líder que se encargue a nivel local, entre otros (Fonseca, 2012; Jara, 2009; O. Quesada, comunicación personal, 10 de abril, 2015; PROSIC, 2009, 2010, 2011).

De acuerdo con O. Quesada (comunicación personal, 17 de febrero, 2014), a febrero del 2014, había instalados y activos $262 \mathrm{CECl}$ a lo largo y ancho del país, cubriendo el $80 \%$ del territorio costarricense (MICITT, 2015). Para el 2015, se confirma el apoyo del Fondo Nacional de Telecomunicaciones para la actualización de los equipos de los $\mathrm{CECl}$ y se trabaja con aquellos $\mathrm{CECl}$ que logren mantenerse activos y funcionando, claramente a iniciativa del grupo comunitario que tiene la responsabilidad del $\mathrm{CECI}$ (O. Quesada, comunicación personal, 10 de abril, 2015).

Además, se incluye el proyecto de los Centros Comunitarios Inteligentes como un proyecto sectorial en el Plan Nacional de Ciencia, Tecnología y Telecomunicaciones 2015-2021. En dicho plan, su objetivo primordial es

"promover la apropiación social y el aprovechamiento de las Tecnologías Digitales, con el fin de disminuir la brecha digital existente en Costa Rica y contribuir a alcanzar metas de desarrollo social, particularmente dentro de poblaciones en condiciones de vulnerabilidad o riesgo social, mediante procesos de aprendizaje, emprendurismo y utilización de servicios en línea". (MICITT, 2015, p. 312)

De acuerdo con datos del MICITT (2014b), en la provincia de Puntarenas hay $37 \mathrm{CECl}$ instalados, de los cuales los siguientes pertenecen al Cantón Central de Puntarenas: Biblioteca Pública de Puntarenas, Jicaral (Universidad Estatal a Distancia [UNED]), Cámara de Pescadores Artesanales de Puntarenas, Barranca, La Gran Chacarita, Pithaya, Biblioteca de la Universidad Estatal a Distancia (UNED) Puntarenas, Universidad Nacional (UNA) Chomes, 
Estación de Biología Marina (EBM) de la Universidad Nacional (UNA), Isla de Chira, Universidad Estatal a Distancia (UNED) Monteverde.

Cabe observar aquí que Puntarenas es la provincia con la mayor cantidad de distritos en la peor condición de acceso a las TIC (MICITT, 2013). Para la presente investigación se trabajó específicamente con los $\mathrm{CECl}$ de: Biblioteca Pública de Puntarenas, Barranca, La Gran Chacarita, Biblioteca UNED Puntarenas y Estación de Biología Marina de la Universidad Nacional (EBM UNA).

\section{Metodología}

Para el desarrollo de la propuesta de modelo se indagó primeramente, en fuentes secundarias, sobre los conceptos alrededor del tema de brecha digital y sobre estrategias existentes y proyectos desarrollados por el Estado para disminuir esta brecha. Seguidamente se investigó sobre el proyecto de los CECI propiamente en fuentes secundarias del MICITT y de la prensa nacional, así como otros documentos relacionados.

Se seleccionaron cinco CECI que se encontraban cercanos a la Sede del Pacífico de la UCR, a un radio máximo de $12 \mathrm{~km}$ de distancia del Centro Universitario, considerando la capacidad operativa, la cercanía al Centro Universitario y los recursos para la investigación. Los cinco CECl seleccionados pertenecen al cantón Central de Puntarenas.

Por medio de instrumentos diseñados al efecto se levantó información de fuentes primarias: entrevistas a cada uno de los encargados de los CECl y al Director del Programa de los CECI del MICITT, visitas de campo a los centros seleccionados; realización de grupos focales en tres comunidades del cantón Central de Puntarenas (Barranca, Fray Casiano de Madrid de Chacarita, El Cocal de Puntarenas), levantamiento de registros de información de la afluencia diaria a los $\mathrm{CECl}$ y otras informaciones relevantes por medio de registro de información en instrumentos diseñados para tal efecto.

Por último se investigó sobre posibilidades existentes a nivel gubernamental de trabajo interinstitucional para el diseño de una propuesta de modelo de trabajo que permita la sostenibilidad de los $\mathrm{CECl}$, por medio de la indagación en leyes y decretos sobre trabajo interinstitucional. Con la información recabada se diseña un modelo educativo y un modelo de sostenibilidad interinstitucional. Ambos modelos se detallan en el apartado Propuesta de modelo.

\section{Resultados del diagnóstico del funcionamiento y administración de los CECl estudiados}

La siguiente información fue obtenida a través de visitas y entrevistas a los encargados de los $\mathrm{CECl}$ realizadas durante el año 2014. Además, se recopiló información diaria de afluencia y otras informaciones relevantes. 


\subsection{Administración y mantenimiento}

La administración del CECl la tiene la persona encargada del CECl. Dicha persona es responsable de la administración y de ofrecer la seguridad y las condiciones de infraestructura y ambiente para el buen funcionamiento del centro. Para el año 2014, los CECl analizados tenían como persona encargada a quien era líder comunal o a quien estaba en la dirección del departamento institucional en donde se encontraba ubicado el CECl.

El mantenimiento del equipo lo realiza el MICITT. Cuando se daña alguna de las computadoras, el encargado del CECI debe coordinar directamente con el MICITT para que envíe a algún técnico a repararla, o enviar la computadora al MICITT. Después de que es reparada se coordina la devolución del equipo al CECI (J. Álvarez, comunicación personal, 4 de febrero, 2014; Farrier, comunicación personal, 3 de febrero, 2014).

En el caso de los CECI ubicados en la UNED y la UNA, los equipos fueron donados por el MICITT a la Universidad (O. Quesada, comunicación personal, 17 de febrero, 2014) con la condición de su custodia y el ofrecimiento del espacio para la capacitación y el uso comunitario como parte del proyecto de los CECl. Los equipos donados pasan a ser activos de la universidad y estas deben encargarse de su mantenimiento. Por su parte, los equipos en los CECI de Barranca, Chacarita y Biblioteca Pública siguen perteneciendo al MICITT.

\subsection{Programas de capacitación}

Los programas de capacitación son: Introducción a la computación, Internet, Word, PowerPoint, Excel, Excel Intermedio y Mantenimiento. Para estos programas se diseñaron manuales que contienen el tema trasversal de Educación Financiera, con el fin de capacitar en principios de finanzas sanas. Cada curso es de 40 horas; al finalizar se les entrega a las personas participantes un certificado que tiene validez en el Servicio Civil.

En los CECI de Barranca, La Gran Chacarita y Biblioteca Pública, la capacitación la lleva a cabo una persona joven del programa Manos a la Obra del Instituto Mixto de Ayuda Social (IMAS). El alumnado sigue la guía o el manual del curso y cuando no entiende algo lo consulta con la persona joven encargada que se encuentra en ese momento en el CECl.

Las personas jóvenes beneficiadas del IMAS reciben ayudas económicas a cambio de realizar su trabajo comunal en el $\mathrm{CECl}$; además, reciben una beca para financiar una carrera parauniversitaria de alta demanda laboral (MICIT, 2012).

Cada estudiante que se inscribe en algún curso de capacitación impartido en el CECl, avanza a su paso realizando los ejercicios del manual del curso. Al finalizar el curso, la persona encargada levanta la lista de quienes concluyeron con el módulo para que el MICITT confeccione los certificados.

Estos tres $\mathrm{CECl}$, son utilizados por la comunidad mayoritariamente para recibir capacitación en TIC. El CECI de la EBM UNA ofrece el espacio para que la comunidad llegue a utilizar el 
Centro. Cuando se inició el proyecto sí se ofrecieron cursos de capacitación, pero la baja en la matrícula hizo que no se volvieran a ofrecer. El Centro es utilizado mayoritariamente por estudiantes colegiales y universitarios de las comunidades vecinas para realizar sus tareas escolares académicas. EL CECI de la UNED Puntarenas tiene un comportamiento similar al CECI de la EBM UNA, ofreciendo actualmente el espacio para uso de la comunidad y, en este caso, la comunidad universitaria de la UNED (estudiantes universitarios).

Al principio del proyecto, el objetivo era instalar laboratorios de cómputo en las diferentes comunidades para que tuvieran acceso a las TIC. Con el transcurso de los años y los cambios en la administración del MICITT, el objetivo del proyecto ha variado. Actualmente se concentra en ofrecer contenidos a la población; para ello se ofrecen cursos de capacitación básica con el fin de habilitar a las personas en el uso de los programas informáticos básicos.

\subsection{Supervisión y control}

Las personas encargadas de los CECI llenan unos informes con datos de su uso al MICITT a través de una plataforma informática que se estaba construyendo al momento de realizar la entrevista (febrero 2014). Cada persona usuaria que llega a un CECl debe llenar un formulario en línea que actualiza automáticamente la información del sistema informático.

Anteriormente las personas encargadas enviaban informes físicos en muy diversos formatos que no facilitaban el análisis ni el levantamiento de estadísticas. Por ello en el 2014 se empezó a capacitar a quienes están a cargo para que ingresen el informe en línea.

En cuanto a los cursos, el MICITT básicamente delega la responsabilidad de la divulgación y promoción de los cursos a ofrecer a quien está a cargo del CECI o a la persona joven del IMAS que atiende el CECI. Quien está a cargo realiza la divulgación a través de la distribución de volantes y la comunicación verbal persona a persona (J. Álvarez, comunicación personal, 4 de febrero, 2014; Farrier, comunicación personal, 3 de febrero, 2014; Largaespada, comunicación personal, 5 de febrero, 2014).

Las personas jóvenes del programa Manos a la Obra del IMAS son instructores y, además, llevan el control de las sesiones de capacitación y el avance de cada estudiante; también pueden colaborar en la divulgación de los cursos. Al concluir la capacitación del curso, la persona que instruye informa al MICITT para que envíe los certificados. EI MICITT se encarga de capacitar a estas personas jóvenes como instructores de los cursos.

\subsection{Afluencia}

Los CECI están abiertos a la comunidad para que reciba capacitación en TIC y para que pueda acceder a los diferentes servicios a través de Internet. No está permitido usar los equipos para jugar.

Las visitas a los CECl ubicados en las Universidades Estatales fueron de personas que requerían de realizar sus tareas académicas personales. Dichos $\mathrm{CECl}$ no ofrecieron ningún curso de capacitación ni tenían programado realizar alguno al momento de la entrevista. En los 
otros tres CECI (Barranca, Chacarita y Biblioteca Pública), la mayoría de las personas que asistieron lo hicieron para recibir algún curso de capacitación, ya sea Word, Power Point o Introducción a la computación.

Por otro lado, el horario en que más visitas recibían era el de 8 a.m. a 12 m.d. en general. Y el rango de edades más común de las personas que visitan los $\mathrm{CECl}$ de Chacarita y Biblioteca Pública era de 36 a 64 años. Al CECl de Barranca lo visitan mayormente jóvenes en el rango de edad de 19 a 35 años. En el CECI de la EBM UNA, la mayoría son jóvenes de 14 a 18 años. No se registró las edades de los visitantes al CECI de la UNED.

\subsection{Inventario de computadoras}

Al momento del levantamiento del inventario (última semana de abril a la primera semana de mayo del 2014), se observó que el CECl de Barranca tenía 6 computadoras HP. Eran nuevas y modernas, con monitores de pantalla plana, procesador Inter Core i5 y 4 GB de memoria RAM.

El CECl de La Gran Chacarita tenía 4 computadoras funcionando con pantallas CRT y 2 computadoras dañadas. Este $\mathrm{CECl}$ no tenía conexión a internet, las razones dadas por la persona encargada fueron: 1- Era muy baja la recepción de la señal de internet en la zona lo que provocaba muchos problemas de conexión. 2- El costo de suscribir el servicio, 3- El nivel de escolaridad de la comunidad donde se encuentra, por lo que se decidió no suscribir el servicio de internet y enfocar el trabajo del CECI hacia la alfabetización digital básica sin entrar al uso de Internet.

La Biblioteca Pública tiene 4 computadoras funcionando con pantallas CRT y 2 computadoras dañadas. EI CECI de la Biblioteca UNED cuenta solo con una computadora funcionando y 4 dañadas. Y el CECI de la EBM UNA cuenta con 6 computadoras funcionando. Este último es el único $\mathrm{CECl}$ que tiene aire acondicionado.

Se aclara que a mayo del 2015, el Lic. Oscar Quesada, director del Proyecto de Centros Comunitarios Inteligentes, informó que se habían aprobado recursos financieros por parte de Fondo Nacional de Telecomunicaciones (FONATEL) para la actualización de los equipos a los CECI (O. Quesada, comunicación personal, 10 de abril, 2015). Pero se iba a trabajar sólo con aquellos $\mathrm{CECl}$ que hubieran demostrado interés y capacidad para la administración y funcionamiento del centro.

\subsection{Diagnóstico de las necesidades comunales}

En general, las necesidades comunitarias giran alrededor de educación a nivel básico principalmente para obtener ingresos económicos inmediatos. El nivel educativo de las personas que acceden a estos centros es bajo; por lo tanto, estas indican requerir cursos que les capaciten para vender productos o servicios y que les puedan deparar ingresos pronto.

La comunidad de Fray Casiano, donde se encuentra ubicado el CECl de La Gran Chacarita, es una de las zonas con mayores problemas sociales, con familias de pobreza y pobreza extrema 
y niveles muy bajos de educación (MIDEPLAN, 2013). A esta le sigue en nivel socioeconómico el de la comunidad de Barranca.

Los CECI ubicados en la Biblioteca Pública de Puntarenas, EBM UNA y Biblioteca de la UNED Puntarenas atienden a una población de nivel socioeconómico un poco superior al de estas comunidades. En el caso de la Biblioteca Pública acceden personas de tercera edad y en el caso de las universidades lo aprovechan estudiantes ya sea escolares, colegiales 0 universitarios.

En cuanto al tema de TIC, los programas de capacitación se han centrado en cursos introductorios de uso del computador, internet y ofimática. Estos se consideran pertinentes debido al nivel educativo de la población que visita estos centros.

Por su parte, como respuesta a la pregunta de sus necesidades educativas en materia de TIC, en los tres grupos focales realizados como parte de esta investigación, las personas solicitaron cursos que les permita obtener beneficios económicos inmediatos como: cursos de costura, tejido, cocina, artesanía, etc. Esto permite observar que estas comunidades no tienen conocimiento del alcance que tiene la educación en TIC, ni cómo pueden aprovecharla.

Asimismo, lo anterior refuerza los resultados de la investigación de Ruiz, Brenes et al. (2012), en la que concluyen que en la zona de Barranca el $70 \%$ de las personas encuestadas indicó que las necesidades más apremiantes son de tipo económico y de seguridad. También indicó que la mayoría de las familias tienen ingresos y niveles de estudios muy bajos.

Aunque no hay estudios específicos de la zona de Fray Casiano de Madrid de Chacarita, donde se encuentra uno de los $\mathrm{CECl}$ analizados, la zona es una de las más deprimidas del cantón Central de Puntarenas, pues ahí se encuentra uno de los precarios de reciente formación con más conflicto por problemas de usurpación. En el mes de febrero de 2015, la policía realizó el desalojo de las familias en estos precarios provocando violencia e inseguridad en la comunidad.

Chacarita, distrito donde se encuentra la comunidad de Fray Casiano de Madrid, se encuentra en una posición de mayor desventaja social según el Índice de Desarrollo Social del 2013 (MIDEPLAN, 2013). El informe sobre este índice, indica que Chacarita se encuentra entre los distritos de desarrollo bajo, y que Barranca y Puntarenas se encuentra entre los distritos de desarrollo medio.

Por lo tanto, el reto es educar en TIC a estas comunidades y ofrecer las experiencias prácticas para aplicar esos conocimientos que les permitan ver su utilidad económica inmediata. Se resalta el hecho de que las personas que asistieron a los grupos focales realizados en La Gran Chacarita y Barranca manifestaron conocer el $\mathrm{CECl}$, aunque no necesariamente con este nombre. Mientras que quienes asistieron al grupo focal del Barrio El Carmen, al que se invitaron personas de las Asociaciones de Desarrollo de las comunidades de Puntarenas Centro, El Cocal y el Barrio El Carmen manifestaron desconocer completamente el proyecto, solo un joven manifestó conocer el CECl de la Cámara de Pescadores Artesanales (este CECl 
no estaba dentro del estudio), pero indicó que hace mucho tiempo que dejó de funcionar porque las computadoras se dañaron y nunca se repararon.

\section{6.}

\section{Propuesta de Modelo}

Del trabajo realizado en esta investigación, se propone crear un modelo interinstitucional y con la comunidad para dar sostenibilidad y funcionamiento a los $\mathrm{CECl}$ con apoyo de FONATEL. Una coordinación interinstitucional y comunitaria se requeriría para mejorar el funcionamiento actual de los $\mathrm{CECI}$, así como para darle la sostenibilidad necesaria para impulsar la reducción de la brecha digital y acercar a las poblaciones siempre excluidas a participar de los beneficios de las TIC y del Gobierno Digital.

\subsection{El modelo educativo y la oferta educativa}

Se propone trabajar el modelo educativo propuesto por Ruiz (2004) -que a continuación se explica- utilizando la infraestructura de los CECI. El modelo educativo, utilizando los CECI, es una propuesta que conjuga innovación, tecnología de información y comunicación, educación y concertación de actores locales. El modelo educativo está propuesto en dos fases, la primera que sería de manera presencial y la segunda de manera virtual.

La oferta educativa de los $\mathrm{CECl}$ será de acuerdo a las necesidades que las propias comunidades prioricen. Esta puede ir desde simples cursos básicos independientes con tutores presenciales o a distancia, a programas más formales a nivel superior contando con la posibilidad de e-learning, educación a distancia o educación virtual.

\subsubsection{Primera Fase}

En una primera fase, el modelo educativo sería con una persona instructora de manera presencial para los cursos de capacitación que se impartan en el CECl; la instrucción puede ser aportada por el Instituto Nacional de Aprendizaje (INA), el Ministerio de Educación Pública (MEP) o las Universidades Estatales por medio de sus Trabajos Comunales Universitarios (TCU). También sería necesario trabajar en una estrategia de apropiación del CECl por parte de la comunidad que permita generar conciencia de la importancia de la utilización de las TIC para sus usuarios y que así se logre que la comunidad aproveche el $\mathrm{CECl}$ en su máxima potencialidad.

Esta primera fase se puede ofrecer de acuerdo a las capacidades institucionales de la zona. En su enfoque más amplio, cada institución educativa aporta, de sus propios recursos, las personas instructoras al proyecto. Así, el MEP, el INA y las Universidades Estatales contribuirían a la sostenibilidad de las capacitaciones en el CECI y al desarrollo de la estrategia de apropiación por parte de las comunidades.

En su enfoque más limitado, considerando que las instituciones educativas no cuentan con los recursos propios para contribuir con el $\mathrm{CECl}$, siempre es posible ofrecer capacitaciones a través de los TCU que deben realizar todos los estudiantes universitarios antes de graduarse. 
Los TCU pueden enfocar su quehacer para contribuir con la sostenibilidad de las capacitaciones en los CECI y desarrollar la estrategia de apropiación comunitaria. En este caso, las unidades académicas de las Universidades Estatales, interesadas en contribuir con los $\mathrm{CECI}$, formularían los proyectos de capacitación en TIC que se impartirían en los CECI.

La primera fase puede ser implementada inmediatamente después de que el grupo comunitario encargado del $\mathrm{CECl}$ coordine con las instituciones correspondientes (MICITT, Ministerio de Economía, Industria y Comercio [MEIC], Ministerio de Agricultura y Ganadería [MAG], Instituto Nacional de Pesca y Acuacultura [INCOPESCA] u otras, y las instituciones educativas) sus necesidades de formación y capacitación.

En esta primera fase, como se indicó anteriormente, es necesario desarrollar una estrategia de apropiación del CECI por parte de la comunidad, la cual deberá ser diseñada. La apropiación requiere generar conciencia en la comunidad para desarrollar estrategias participativas para que el ciudadano se apropie del CECI, lo cual implica esfuerzos de educación formal y no formal para el entendimiento de las TIC y su importancia para el desarrollo. Un ciudadano que se apropie del CECl podrá sacar provecho al utilizarlo para su propio desarrollo productivo, académico u otro (MICITT, 2015).

De acuerdo a las necesidades diagnosticadas en los $\mathrm{CECl}$, se coordina la apertura de los programas educativos para atenderlas a través de las instituciones educativas correspondientes y la utilización de la plataforma tecnológica del CECl. Es posible la apertura de programas de educación formal del Ministerio de Educación para ofrecer el bachillerato a las personas que no han concluido la educación secundaria, así como cursos de capacitación en temas de gestión empresarial a los microempresarios o de uso de sistemas satelitales para la ubicación de bancos de peces a los pescadores, o capacitación en la búsqueda, acceso y uso de información agropecuaria para los agricultores, entre muchas otras posibilidades.

\subsubsection{Segunda Fase}

Para la segunda fase es necesario haber trabajado fuertemente en la apropiación del CECI por parte de la comunidad, así como tener una base comunitaria con la alfabetización digital mínima, que haga posible el uso de las TIC por parte de los individuos de la comunidad para su propio beneficio (productivo, educativo, personal). Sin esto, la segunda fase no tendría mayor impacto.

Para la segunda fase se propone desarrollar un modelo educativo que comprenda un sistema de comunicación que sustituye la interacción personal docente-estudiante por la interacción con una gran variedad de recursos didácticos, diseñados para el autoaprendizaje. La tecnología informática ofrece el acceso a ambientes de aprendizaje donde el alumnado interactúa con contenidos, bases de datos, estrategias de enseñanza y aprendizaje en el momento que más le conviene.

Para esta segunda fase se debe indagar sobre plataformas educativas tecnológicas para impartir cursos virtuales, metodologías de enseñanza y aprendizaje virtual, estrategias de asesoría y retroalimentación permanente al estudiante y mecanismos de evaluación y control que aseguren la calidad de la instrucción, similar a los cursos online, masivos y abiertos 
(MOOC por sus iniciales en inglés) que actualmente se ofrecen en numerosas instituciones y organizaciones públicas y privadas (Marauri, 2014).

Como lo indica Ruiz (2004):

el aprendizaje no consiste sólo en organizar y procesar información, sino construir conocimientos con base en la experiencia de cada persona. Para ello se invita a los participantes a seleccionar la información más adecuada para sus necesidades inmediatas, y a integrarlas a su vida diaria. (p. 12)

La idea de la segunda fase es ayudar a quienes son estudiantes para que construyan sus esquemas de conocimiento. El modelo se enfoca a apoyar al alumnado para que desarrolle su capacidad de aprendizaje auto dirigido. La intención es formar personas emprendedoras, capaces de aprender lo que desean, con autoconfianza y autoestima, como resultado del proceso educativo.

Para la segunda fase se requiere recursos financieros para la investigación y desarrollo de la plataforma tecnológica, metodologías didácticas, evaluativas, supervisión y control, así como el desarrollo del sitio web, además del mantenimiento y sostenibilidad de la infraestructura que se desarrolle. Esta segunda fase, que soporta el modelo educativo virtual y autónomo, sólo podría ser desarrollada con recursos financieros extraordinarios, los cuales podrían ser aportados por FONATEL, si se desea una plataforma propia.

Sin embargo, en la red ya existe oferta de educación de todos los niveles y para todo tipo de necesidades, por lo que es posible utilizar estas alternativas de enseñanza-aprendizaje para estos efectos. Las posibilidades de acceso a la información y a los procesos educativos amplían las posibilidades de aprendizaje, desarrollo de destrezas y competencias con posibilidad de desarrollar proyectos productivos o para acceder a mejores empleos, contribuyendo así a la movilidad social y a mejorar la calidad de vida.

\subsection{Modelo de sostenibilidad interinstitucional}

En mayo de 2010 se promulgó la Ley 8801 Ley General de Transferencia de Competencias (2010) del Poder Ejecutivo a las Municipalidades. Dicha ley crea los Consejos Cantonales de Coordinación Interinstitucional $(\mathrm{CCCl})$. El reglamento a esta Ley contiene un capítulo que detalla el funcionamiento de estos consejos, integrados por todo ente público que desarrolle actividades en la localidad y sociedades anónimas-empresas públicas (Decreto Ejecutivo, 2010). Este puede ser el espacio para atender la sostenibilidad de los CECI mediante el trabajo interinstitucional, con el grupo comunitario y con el encargado del $\mathrm{CECl}$.

Se propone el consejo regional, en este caso sería el CCCI en conjunto con el comité comunal (que puede ser la asociación de desarrollo comunal), como encargado de apoyar el desarrollo y funcionamiento del CECI. Cada una de las instituciones del Estado que forman parte del CCCI tiene sus funciones y recursos financieros establecidos. El trabajo colaborativo interinstitucional dentro del $\mathrm{CCCl}$ para apoyar el funcionamiento del $\mathrm{CECl}$ exige que cada institución cumpla sus 
propias funciones institucionales. El CECl ofrece el espacio para que cada institución pueda ampliar su cobertura atendiendo poblaciones vulnerables y siempre excluidas.

El CCCI se encargaría, entre otros, de:

- impulsar el proyecto educativo,

- iniciar proyectos productivos locales que generen recursos para el desarrollo comunitario y la sostenibilidad del CECI,

- ayudar al comité comunal a establecer vínculos con organismos públicos y privados,

- supervisar el cumplimiento del trabajo de cada una de las instituciones con respecto al proyecto.

Se propone que la comunidad mediante un grupo organizado (comité comunal o asociación de desarrollo) se encargue de buscar el espacio para la instalación de los CECI, así como de dotar de los servicios básicos para su funcionamiento (luz, agua, telecomunicación, seguridad). Además debe aportar una persona encargada responsable de atender el centro.

La persona encargada, en conjunto con el MICITT y con las instituciones correspondientes en materia de educación, tendría la tarea de identificar las necesidades de capacitación y coordinar para el diseño de un plan de capacitación con las necesidades identificadas y priorizadas, así como la administración del CECl y su divulgación. Debidamente capacitado por el MICITT, esta persona encargada también puede ser quien instruya en los cursos básicos de alfabetización digital.

Con respecto a la seguridad del $\mathrm{CECl}$, el Ministerio de Seguridad Pública (MSP) podría coordinar acciones para el cuido de estos centros, considerando que muchos de estos se ubican en comunidades de riesgo social. EI MSP cuenta con recursos para mantener la seguridad en las comunidades, por lo que no sería necesario recursos extraordinarios para mantener la seguridad de las comunidades donde se encuentra ubicado un $\mathrm{CECl}$, pues es parte de sus funciones. Es cuestión de planificar mejor los recursos y las rutas de patrullaje.

En cuanto a la divulgación, se considera muy importante diseñar una estrategia de información y comunicación que divulgue sobre los cursos y servicios del CECl, y además motive a los pobladores a acercarse y desear capacitarse en materia de TIC, de tal forma que las comunidades se apropien del $\mathrm{CECl}$ y lo aprovechen al máximo para realizar sus procesos comerciales, productivos, financieros o académicos. El diseño y la implementación de la estrategia de divulgación, comunicación y apropiación, podrían ser realizados con apoyo de recursos financieros de FONATEL.

EI MICITT, como ente director del proyecto, se responsabilizaría de dotar los equipos tecnológicos, mobiliario, instalación, mantenimiento preventivo y correctivo así como de la actualización del equipo y programas utilizados, y de dar capacitación a las personas encargadas para su funcionamiento. Además le correspondería definir los protocolos de organización y administración de los CECl. También, en conjunto con el comité comunal, debe apoyar a este en el diagnóstico de sus necesidades, en la promoción y la generación de las alianzas con los actores locales. Asimismo, el MICITT, en conjunto con el grupo comunitario, 
coordinaría con el Instituto Costarricense de Electricidad (ICE) los asuntos de infraestructura tecnológica necesaria para que el $\mathrm{CECI}$ cuente con las últimas TIC que permitan ofrecer sus servicios (académicos, comerciales, productivos, financieros) de manera eficiente.

El Sistema Nacional de Bibliotecas (SINABI) colaboraría como lo ha estado haciendo; esto es, continuaría ofreciendo el espacio y el personal para implementar el CECl y sus programas educativos en sus instalaciones.

Las Universidades Públicas, por medio de sus TCU, podrían desarrollar proyectos de Trabajo Comunal en los cuales su estudiantado realice sus horas comunales participando en los CECI desarrollando actividades según un plan que debe plasmar cada $\mathrm{CECI}$ de acuerdo con las necesidades de capacitación diagnosticadas en su comunidad. Las Universidades Públicas apoyarían de esta manera el proyecto educativo, permitiendo a estudiantes y docentes relacionarse e involucrarse con las necesidades comunales, sensibilizándoles en la problemática social de las comunidades en la que se insertan y formándoles en nuevas herramientas y metodologías para atender poblaciones con otras necesidades educativas.

El INA aportaría si las necesidades son en materia de educación técnica y el MEP si las necesidades son en educación formal primaria y secundaria. Estas dos instituciones, MEP e INA, podrían también coordinar acciones formativas con las Universidades Estatales, en caso de que no cuenten con los recursos propiamente para realizar su labor de formación a las poblaciones de estas comunidades marginales.

Las otras instituciones públicas podrían capacitar en el uso y manejo de la tecnología para la comunicación e información con sus clientes, así como la atención del servicio a sus usuarios. Por ejemplo, la banca podría capacitar a los usuarios en el uso de sus plataformas web para todo lo relacionado con pago de servicios, préstamos, solicitud de créditos, banca de desarrollo. EI MAG podría capacitar en el uso de la tecnología para informar a agricultores y agricultoras en el manejo de la información agropecuaria. El INCOPESCA podría capacitar a las personas pescadoras en el uso de sistemas satelitales para localizar bancos de peces. El MEIC podría capacitar a las personas micro y pequeño empresarias en el uso de la tecnología para desarrollar el comercio electrónico y para relacionarse con el Estado como proveedoras. El Ministerio de Hacienda $(\mathrm{MH})$ podría capacitar a las personas micro y pequeño empresarias, así como a profesionales liberales en el uso de sus sistemas de tributación en línea, entre otros.

Tanto el Ministerio de Trabajo y Seguridad Social (MTSS) como el IMAS podrían ser entes colaboradores con sus programas como Empléate y Manos a la Obra; dichos programas sociales aportan un subsidio a la persona beneficiaria a cambio de algún trabajo comunal que podría ser realizado en el CECI. La Dirección Nacional de Desarrollo Comunal (DINADECO) apoyaría a las asociaciones de desarrollo en la labor que realizarían en la administración de los $\mathrm{CECl}$ y en el diagnóstico de necesidades. Y las municipalidades como coordinadoras de los $\mathrm{CCCl}$ serían las que velarían para que cada una de las instituciones realice las tareas que se le encomiende relacionadas con los CECl. 
- De esta manera, el CECI sería no solo un centro de capacitación sino también de información y comunicación con las instituciones del Estado; asimismo, sería un centro para realizar transacciones electrónicas comerciales, tributarias, crediticias, etc.

Por último, los recursos financieros necesarios para llevar a cabo la propuesta puede ser aportada por el FONATEL. Todo el proyecto de CECl debería incluirse como un proyecto prioritario dentro del programa de Gobierno Digital, puesto que es una de las estrategias implementadas para disminuir la brecha digital. Un gobierno líder en gobernanza digital debe contar con una ciudadanía capaz de aprovechar las potencialidades que ofrecen las TIC para mejorar su calidad de vida.

Finalmente, el Ministerio de Planificación y Política Económica (MIDEPLAN), como ente del gobierno que asesora la implementación de los $\mathrm{CCCl}$, debe velar para que los acuerdos en este Consejo se cumplan con la colaboración de todas las entidades involucradas. La Figura 1 muestra la propuesta de sostenibilidad planteada. Dicha propuesta es posible en los cantones donde el $\mathrm{CCCl}$ funciona adecuadamente y han tomado con seriedad el trabajo y los acuerdos que se realizan en este consejo.

\section{Figura 1}

Propuesta de modelo

\begin{tabular}{|c|c|c|}
\hline \multicolumn{3}{|c|}{ Centro Comunitario Inteligente (CECI) } \\
\hline \multicolumn{3}{|c|}{ Encargado del CECl } \\
\hline $\begin{array}{l}\text { Administración, Seguridad, } \\
\text { Mantenimiento, Divulgación }\end{array}$ & $\begin{array}{l}\text { Educación formal, no } \\
\text { formal }\end{array}$ & Infraestructura tecnológica \\
\hline \multirow{3}{*}{$\begin{array}{c}\text { Grupo Comunitario, MICITT, } \\
\text { Ministerio de Seguridad, } \\
\text { DINADECO }\end{array}$} & $\begin{array}{c}\text { Estudiantes de TCU, } \\
\text { personas beneficiarias de } \\
\text { IMAS, MTSS. }\end{array}$ & \multirow[t]{3}{*}{$\begin{array}{l}\text { MICITT, ICE, Grupo } \\
\text { Comunitario }\end{array}$} \\
\hline & $\begin{array}{l}\text { MICITT, Grupo Comunitario, } \\
\text { Universidades Estatales, } \\
\text { MEP, INA, MAG, MEIC, } \\
\text { SINABI, INCOPESCA, banca } \\
\text { estatal. }\end{array}$ & \\
\hline & $\begin{array}{l}\text { MTSS, IMAS, DINADECO, } \\
\text { Municipalidad }\end{array}$ & \\
\hline \multicolumn{3}{|c|}{ Consejo Cantonal de Coordinación Interinstitucional (CCCI) } \\
\hline \multicolumn{3}{|c|}{ FONATEL, Gobierno Digital, MIDEPLAN } \\
\hline
\end{tabular}

Fuente: Elaboración propia con datos de la investigación. 
Al existir en el país 81 cantones, cada uno de ellos con diferencias en cuanto a necesidades y dinámicas de funcionamiento, es posible que el trabajo colaborativo interinstitucional no funcione bien en algunos de los cantones. Para estos casos, el modelo contempla la figura de una persona encargado del $\mathrm{CECl}$, la cual puede ser aportada por la colaboración de las instituciones públicas y privadas que forman parte del $\mathrm{CCCl}$ o que el grupo comunitario logre los recursos necesarios para aportar esta persona encargada. Por ejemplo, puede ser que el primer año quien se encargue del CECl sea un aporte de la asociación de desarrollo comunal; el segundo, de alguna institución; y el tercer año, de alguna empresa privada, etc. También podría ser un aporte entre varias entidades, cada una dando recursos parciales para cubrir el gasto.

Esta persona encargada del $\mathrm{CECI}$, debidamente capacitada por el MICITT, sería la persona que coordinaría y realizaría las gestiones necesarias ante las demás instituciones para lograr realizar la tarea encomendada al $\mathrm{CECl}$. Es decir, gestionaría ante la institución que corresponda: la apertura de cursos de capacitación en materia de TIC y otros, el mantenimiento del laboratorio, la seguridad, la actualización del hardware y software, la divulgación y la promoción del CECl. Además, dicha persona puede ser instructora de los cursos de alfabetización básica.

La figura de la persona encargada ha sido implementada en algunos de los CECI más exitosos (O. Quesada, comunicación personal, 10 de abril, 2015). Sin que todavía exista la plataforma interinstitucional de colaboración, estos $\mathrm{CECI}$ han funcionado de manera excelente debido a que tienen un grupo comunitario que se ha apropiado del $\mathrm{CECl}$ y ha conseguido mantener la persona encargada. En estos $\mathrm{CECl}$ se ha logrado una muy buena convocatoria a las capacitaciones en alfabetización digital de la comunidad.

En el funcionamiento actual de los $\mathrm{CECl}$ estudiados, la figura de la persona encargada recae en quien es director o directora de la institución donde se aloja el $\mathrm{CECl}$ o en quien es líder del grupo comunitario. La persona encargada propuesta en el modelo es una que no tiene otras funciones más que las propias de administrar el funcionamiento del centro, como se indicó anteriormente. La idea es conseguir los recursos para tener esta persona a cargo permanentemente.

La experiencia desarrollada por los CECI durante toda su vigencia ha formado y capacitado a jóvenes del programa Manos a la Obra del IMAS como instructores de la alfabetización básica. Estas personas jóvenes fueron beneficiarias que provenían de comunidades deprimidas. Se propone que las personas jóvenes que trabajaron en los CECI de sus comunidades, que hayan demostrado su capacidad de liderazgo e instrucción así como compromiso con la comunidad, sean las primeras en considerarse para el puesto de encargado del CECI. Dicho trabajo deberá ser remunerado con el aporte colaborativo de las instituciones públicas, privadas 0 comunitarias, como se indicó anteriormente. 
El proyecto de Centros Comunitarios Inteligentes fue implementado a partir del 2007 como resultado del Plan Nacional de Desarrollo 2006-2010, finalizando el año 2010 con $278 \mathrm{CECl}$ (MIDEPLAN, 2011). A febrero del 2014, había 262 CECl activos. El proyecto permitió desarrollar e implementar una estructura administrativa y organizativa de una estrategia para disminuir la brecha digital, además de ofrecer la experiencia para mejorar el proyecto en los próximos años.

De acuerdo con la investigación, se identifican debilidades del proyecto:

- Escasez de recursos presupuestarios suficientes para el mantenimiento y actualización de la infraestructura tecnológica para un servicio del CECl deseable.

- Pocos recursos para realizar una labor de supervisión y control adecuada del funcionamiento y administración de los CECI desde la coordinación central.

- Falta una estrategia de divulgación, información y comunicación que además de divulgar motive a las personas de la comunidad a acercarse al $\mathrm{CECl}$, conocer de sus servicios, apropiarse del espacio y aprovecharlo para sus necesidades personales, académicas, comerciales, productivas y/o financieras.

- Necesidad de una oferta educativa básica y otra oferta adaptada a las necesidades de la comunidad que permita a las personas beneficiarias capacitarse y ver resultados prácticos y de utilidad económica inmediata, esto debido a que los CECI se encuentran en zonas marginales de alto riesgo social, con poblaciones de muy baja escolaridad y de muy baja condición socioeconómica.

- La eficacia mayor de los CECl se da en aquellascuya labor es administrada por los encargados de grupos comunitarios, y el ubicado en la Biblioteca Pública. Los CECI ubicados en Universidades Públicas son más utilizados por estudiantes para tareas escolares, colegiales o universitarias, que en cursos de capacitación a la comunidad; por otro lado, la actualización de los equipos llega a ser parte de la responsabilidad de la propia universidad que en ocasiones no cuentan con recursos para su mantenimiento.

Para resolver estos aspectos, se propone una modelo de sostenibilidad interinstitucional con el grupo comunitario encargado del $\mathrm{CECl}$ y con recursos financieros de FONATEL descritos con detalle en este trabajo. Sin embargo, si los acuerdos interinstitucionales no se logran alcanzar o si el Consejo Cantonal de Coordinación Interinstitucional no funciona adecuadamente, es posible el funcionamiento de estos centros de acuerdo al modelo propuesto por la presencia de la persona encargada. Para esto se requiere que la comunidad interesada logre el apoyo para tener una persona encargada responsable del centro que imparta los cursos o coordine con las instituciones correspondientes para ofrecer las capacitaciones. Además, esta persona encargado sería responsable de la administración y funcionamiento del $\mathrm{CECl}$, asesorada estrechamente por el MICITT y apoyada por el grupo comunitario, como se explicó en detalle.

Lo más reciente que se ha realizado con respecto a los $\mathrm{CECl}$ es su inclusión como un proyecto sectorial en el Plan Nacional de Ciencia, Tecnología y Telecomunicaciones 2015-2021. Si este plan se logra, sería un gran avance para el país en su meta de disminuir la brecha digital, y se estaría resolviendo varias de las debilidades anotadas al inicio de estas conclusiones. Por 
"último, el hecho de incluir el proyecto de Centros Comunitarios Inteligentes 2.0 entre los proyectos a ejecutarse en el Plan Nacional de Desarrollo 2015-2018 (MIDEPLAN, 2014) es un excelente comienzo para alcanzar el objetivo de estos Centros que contribuya a disminuir la brecha digital.

\section{Referencias}

Asociación Latinoamericana de Integración. (2003). La brecha digital y sus repercusiones en los países miembros de la ALADI. Recuperado de http://www.aladi.org/nsfaladi/estudios.nsf/decd25d818b0d76c032567da0062fec1 /169f2e26bfc7a23c03256d74004d6c5f/\$FILE/157Rev1.pdf

Decreto Ejecutivo No.36004-PLAN. (2010). Recuperado de http://www.mopt.go.cr:10039/portal/Gestion\%20Municipal/Documentos/Normativ a/Reglamento_LeyGeneral_TransferenciaCompetencias.pdf

Fonseca, P. (14 de abril de 2012). Acceso a Internet es un derecho lejano para vecinos de costas ticas. La Nación, p. 18A.

Fundación Orange. (2004). IV Informe anual sobre el desarrollo de la Sociedad de la Información en España. Madrid: Autor.

Hilbert, M. R. (2001). From industrial economics to digital economics. Santiago, Chile: CEPAL.

Instituto Nacional de Estadística y Censos. (2014). Encuesta Nacional de Hogares. Recuperado de http://www.inec.go.cr/enaho/result/tics.aspx

Jara, E. (2009). Análisis de los Centros Comunitarios Inteligentes como una política pública para disminuir la brecha digital en Costa Rica. Trabajo presentado en la Segunda Conferencia Internacional sobre Brecha Digital e Inclusión Social, Leganés, Madrid.

Lara, J. (31 de julio de 2014). 88\% de accesos a Internet en el país se hacen desde un celular. La Nación, p. 17A.

Ley 8801 General de Transferencia de Competencias del Poder Ejecutivo a las Municipalidades. (04 de mayo de 2010). La Gaceta Digital Diario Oficial, (85). Recuperado de http://www.gaceta.go.cr/pub/2010/05/04/ALCA7_04_05_2010.html

Marauri, P. M. (2014). La figura de los facilitadores en $\overline{l o s} \bar{C}$ ursos Online Masivos y Abiertos (COMA / MOOC): nuevo rol profesional para los entornos educativos en abierto. RIED. Revista Iberoamericana de Educación a Distancia, 17(1), 35-67.

Ministerio de Ciencia y Tecnología. (2012). Proyecto Acceso Digital y empleabilidad: CECI 2-Manos a la Obra. Recuperado de http://ceci14.webnode.es/ceci-manosa-la-obra/descripcion-del-proyecto/

Ministerio de Ciencia, Tecnología y Telecomunicaciones. (2014b). CECI Puntarenas. Recuperado http://ceci14.webnode.es/ceci-por-provincia/puntarenas/

Ministerio de Ciencia, Tecnología y Telecomunicaciones. (2015). Plan Nacional de Ciencia, Tecnología e Innovación 2015-2021. San José, Costa Rica: Ministerio de Ciencia, Tecnología y Telecomunicaciones.

Ministerio de Ciencia, Tecnología y Telecomunicaciones. (2013). Ranking distrital de acceso a las tecnologías de información y comunicación. Recuperado de 
http://www.elfinancierocr.com/tecnologia/Ranking-Distrital-Final-

Ajustado_ELFFIL20130710_0001.pdf

Ministerio de Ciencia, Tecnología y Telecomunicaciones. (2014a). CECl Descripción del proyecto. Recuperado http://ceci14.webnode.es/descripcion-del-proyecto/

Ministerio de Planificación y Política Económica. (2014). Plan Nacional de Desarrollo 2015-2018 "Alberto Cañas Escalante". San José: Autor.

Ministerio de Planificación y Política Económica. (2011). Plan Nacional de Desarrollo: Informe de Evaluación Anual 2010. Recuperado de http://documentos.mideplan.go.cr/alfresco/d/d/workspace/SpacesStore/31d2f1e6 -709c-419f-809e-cf98c363d90e/Informe_Evaluacion_PND_\%202010.pdf

Ministerio de Planificación y Política Económica. (2013). Índice de Desarrollo Social 2013, Costa Rica. San José, Costa Rica: Autor.

Monge, R. y Hewitt, J. (2004). Tecnologías de la Información y las Comunicaciones (TIC) y el futuro de Costa Rica. San José: Fundación CAATEC.

Muñoz, D., y Nicaragua, R. (2014). Un acercamiento a la brecha digital en Costa Rica. e-Ciencias de la Información, 4(1), 30.

Programa Sociedad de la Información y el Conocimiento. (2006). Informe 2006 Hacia la Sociedad de la Información y el Conocimiento en Costa Rica. San José: Universidad de Costa Rica. Recuperado de http://www.prosic.ucr.ac.cr/materiales/informes/informe-2006

Programa Sociedad de la Información y el Conocimiento. (2009). Informe 2009 Hacia la Sociedad de la Información y el Conocimiento en Costa Rica. San José: Universidad de Costa Rica.

Programa Sociedad de la Información y el Conocimiento. (2010). Informe 2010 Hacia la Sociedad de la Información y el Conocimiento. San José: Universidad de Costa Rica. Recuperado de http://www.prosic.ucr.ac.cr/materiales/informes/informe2010

Programa Sociedad de la Información y el Conocimiento. (2011). Informe 2011 Hacia una Sociedad de la Información y el Conocimiento en Costa Rica. Recuperado de http://www.prosic.ucr.ac.cr/materiales/informes/informe-2011

Ruiz, L. (2004). Red de Centro Comunitarios de Aprendizaje: una estrategia educativa para apoyar la inclusión social y el desarrollo de agentes de cambio. Trabajo presentado en el Primer Congreso Virtual Latinoamericano de Educación a Distancia.

Ruiz, N., Brenes, A., Vega, L., Vargas, R., Mora, A., Padilla, C., ... y Chavarría, C. (2012). Estudio de opinión para evaluar el impacto o el aporte de la Universidad de Costa Rica, Sede del Pacífico en Barranca. Puntarenas, Costa Rica: Universidad de Costa Rica.

Serrano Santoyo, A. y Martínez, E. (2003). Brecha digital: mitos y realidades. México: Editorial Universitaria de la Universidad Autónoma de Baja California. Recuperado de http://www.labrechadigital.org/labrecha/LaBrechaDigital_MitosyRealidades.pdf 


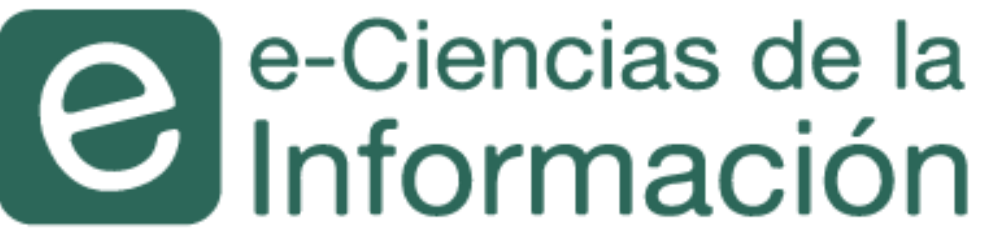

¿Desea publicar su trabajo? Ingrese aquí

O escríbanos a la siguiente dirección: revista.ebci@ucr.ac.cr

(이()(2)

\section{1}

Origen: respuesta a una necesidad

En el año 2011, la Escuela de

Bibliotecología y Ciencias de la Información (EBCI) de la Universidad de Costa Rica (UCR) reconoció la importancia de crear nuevas y mejores alternativas para difusión de la investigación. e-Ciencias de la Información es la respuesta a un contexto actual marcado por una mayor apertura, flexibilidad, y rigurosidad en la verificación de los datos y su procesamiento.

\section{3}

Revista de la UCR

e-Ciencias de la Información es una revista científica que aborda las nuevas temáticas de desarrollo e investigación en las Ciencias de la Información, en el ámbito nacional e internacional. Así, colabora significativamente en el progreso de esta disciplina. Por sus parámetros de calidad, pertenece al grupo de las revistas más importantes de la UCR y se encuentra ampliamente indizada en los importantes catálogos.

\section{En la actualidad}

\section{Posicionamiento internacional}

La revista admite trabajos en las siguientes áreas, siempre que se relacionen directamente con las Ciencias de la Información:

Bibliotecología, Documentación, Tecnologías de la Información y la Comunicación, Investigación, Análisis Estadísticos y Bibliometría, Archivística, Sistemas de Información, Informática, Comunicación y Biblioteas Escolares.

\section{latindex DOAJ $0_{\text {SHERPA/Romeo }}^{0}$ Dialnet e-revist@s}

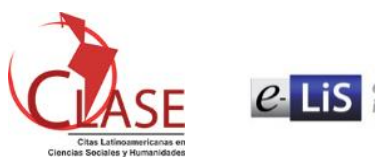

e-prints in library \&
information science

\section{INF 3 B I L A A Open Science Directory \\ OU ULRICHSWEB'm \\ Google}

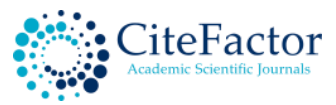

